\title{
Distances to nearby galaxies around IC $342^{\star, \star \star}$
}

\author{
I. D. Karachentsev ${ }^{1}$, M. E. Sharina ${ }^{1,4}$, A. E. Dolphin ${ }^{2}$, and E. K. Grebel ${ }^{3}$ \\ 1 Special Astrophysical Observatory, Russian Academy of Sciences, N. Arkhyz, KChR 369167, Russia \\ 2 Kitt Peak National Observatory, National Optical Astronomy Observatories, PO Box 26732, Tucson, AZ 85726, USA \\ 3 Max-Planck-Institut für Astronomie, Königstuhl 17, 69117 Heidelberg, Germany \\ ${ }^{4}$ Isaac Newton Institute, Chile, SAO Branch, Russia
}

Received 24 April 2003 / Accepted 10 June 2003

\begin{abstract}
We present an analysis of Hubble Space Telescope/WFPC2 images of six nearby galaxies in the projected vicinity of IC 342: Cas dSph, KK 35, UGCA 86, Cam A, NGC 1560, and Cam B. We derive distances to five of them from the luminosity of the tip of the red giant branch stars with a typical accuracy of $\sim 10 \%$. The galaxy distances are $0.79 \mathrm{Mpc}(\mathrm{Cas} \mathrm{dSph}$ ), 3.16 Mpc (KK 35), 3.93 Mpc (Cam A), 3.45 Mpc (NGC 1560), and 3.34 Mpc (Cam B). Two other observed galaxies, MB 2 and Cam C $=$ KK 26, turn out to be Galactic cirrus and a Galactic H II region, respectively. Cas dSph belongs to the Local Group and is a companion of M31. Combining our data with literature results, we find that there are seven dwarf galaxies associated with the giant spiral galaxy IC 342. This group is characterized by an average distance of $(3.28 \pm 0.15)$ Mpc, an average radial velocity of $(229 \pm 23) \mathrm{km} \mathrm{s}^{-1}$, a projected radius of $322 \mathrm{kpc}$, a radial velocity dispersion of $60 \mathrm{~km} \mathrm{~s}^{-1}$, and a total blue luminosity of $3.43 \times 10^{10} L_{\odot}$. The derived virial and orbital mass-to-luminosity ratios are 20 and $28 M_{\odot} / L_{\odot}$, respectively. The galaxy group around Maffei 1 has so far a less reliable distance of $\sim 3.0 \mathrm{Mpc}$ and an average radial velocity of $(309 \pm 22) \mathrm{km} \mathrm{s}^{-1}$. This group consists of eight galaxies and is characterized by a projected radius of $112 \mathrm{kpc}$, a radial velocity dispersion of $59 \mathrm{~km} \mathrm{~s}^{-1}$, and a total blue luminosity of $2.97 \times 10^{10} L_{\odot}$. For the Maffei group we estimate mass-to-light ratios of $M_{\mathrm{vir}} / L_{B}=16$ and $M_{\text {orb }} / L_{B}=5$ in solar units. The sum of the virial $\left(119 \times 10^{10} M_{\odot}\right)$ and orbital $\left(109 \times 10^{10} M_{\odot}\right)$ masses of both groups agree well with their total mass, $(107 \pm 33) 10^{10} M_{\odot}$, derived from the radius of the "zero velocity surface", $R_{0}=(0.9 \pm 0.1) \mathrm{Mpc}$, which separates the IC 342/Maffei complex from the Hubble flow.
\end{abstract}

Key words. galaxies: dwarf - galaxies: distances and redshifts - galaxies: kinematics and dynamics - galaxies: individual: IC 342

\section{Introduction}

Among nearby galaxy groups that are comparable with the Local Group (LG) in their integrated luminosity and number of galaxies, the group of galaxies around IC 342, Maffei 1, and Maffei 2 is one of the nearest ones. Since the distances to the dominant members of this group were only poorly known and since some of these galaxies were believed to be very close to the LG, Zheng et al. (1991), Valtonen et al. (1993), and Peebles (1994) have suggested that the IC 342/Maffei complex might affect essentially the trajectories of galaxies in the LG. However, in spite of its "strategic" disposition with respect to us, the IC 342/Maffei group remained a less studied group because of strong extinction by dust clouds in the

Send offprint requests to: I. D. Karachentsev,

e-mail: ikar@luna.sao.ru

* Based on observations made with the NASA/ESA Hubble Space Telescope. The Space Telescope Science Institute is operated by the Association of Universities for Research in Astronomy, Inc. under NASA contract NAS 5-26555.

$\star \star$ Figure A.1 is only available in electronic form at http://www. edpsciences.org
Galactic plane. Heliocentric velocities of the group members are concentrated in the range of $[-100,+150] \mathrm{km} \mathrm{s}^{-1}$, where the emission of Galactic neutral hydrogen makes radial velocity measurements extremely difficult. Most of the galaxies of the group were only discovered during the last decade through special searches in the H I line (Kraan-Korteweg et al. 1994; Huchtmeier et al. 1995; Huchtmeier \& van Driel 1996; Huchtmeier et al. 1997), as well as in the infrared and optical (McCall \& Buta 1995; Karachentseva \& Karachentsev 1998; Karachentsev et al. 2001). The general properties of the IC 342/Maffei group have been described by Krismer et al. (1995), Karachentsev et al. (1997), Buta \& McCall (1999), and Huchtmeier et al. (2000).

At the present time, studying structure and kinematics of the IC 342/Maffei complex is restrained by a lack of data on distances to its members. Only recently, accurate distances have been measured to the three dwarf galaxies: UGCA 105 , $\mathrm{KKH} \mathrm{5,} \mathrm{and} \mathrm{KKH} 34$ from the luminosity of their red giant stars (Karachentsev et al. 2002a, 2003a). The distance to the brightest group member, IC 342, was determined by Saha et al. (2002) from the $P-L$ relation for Cepheids. 


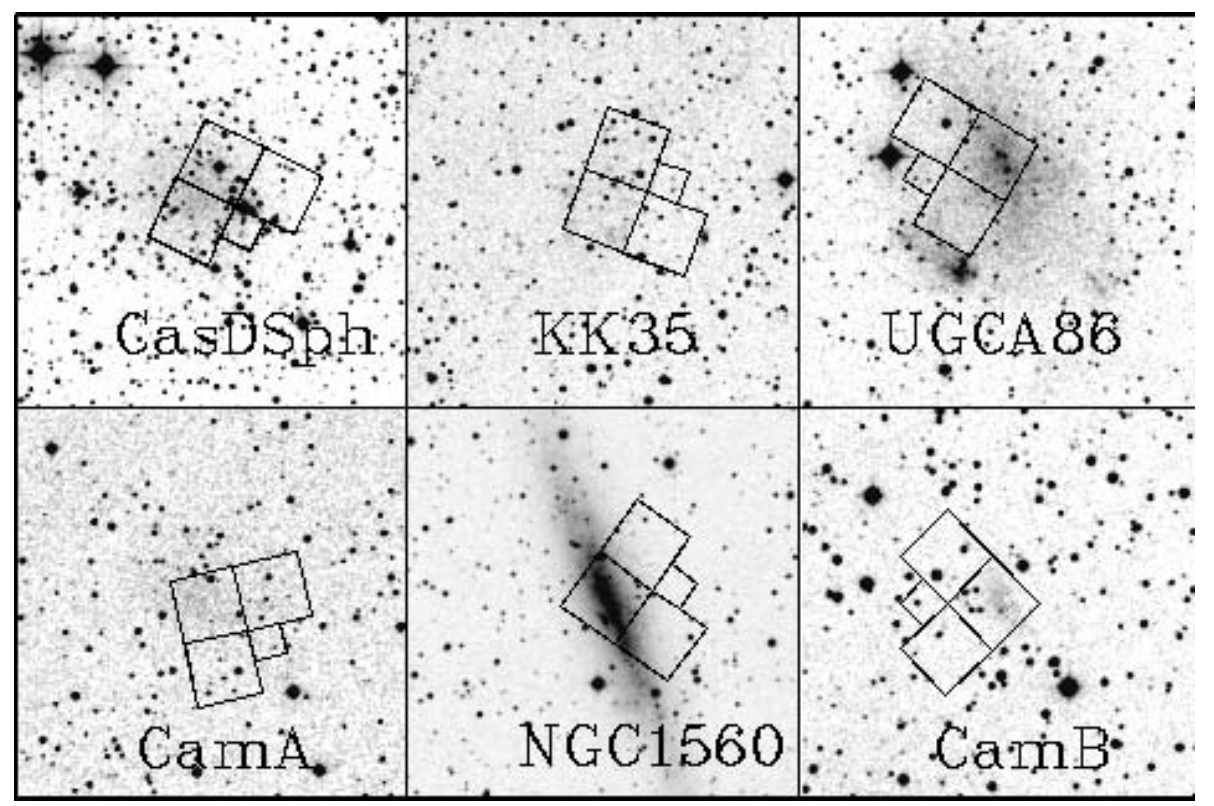

Fig. 1. Digital Sky Survey images of six nearby galaxies in wide vicinity of IC 342 . The field size is $8^{\prime}$, North is up and East is left. The HST WFPC2 footprints are superimposed.

Fingerhut et al. (2003) estimated the distance to the elliptical galaxy Maffei 1 using its central velocity dispersion and $I$ band photometry. In the present paper we determine new distances to five more galaxies in the vicinity of IC 342 based on their tip of red giant branch (TRGB) derived from the Hubble Space Telescope (HST) images. Together with the earlier published data, this gives us the opportunity to analyze the structure of the group and to determine its total mass for the first time.

\section{WFPC2 photometry}

Images of six galaxies were obtained with the Wide Field and Planetary Camera (WFPC2) aboard the Hubble Space Telescope between July 12, 1999 and February 28, 2000 as part of our HST snapshot survey of nearby galaxy candidates (Seitzer et al. 1999; Grebel et al. 2000). The galaxies were observed with 600-s exposures taken in the F606W and F814W filters for each object. Digital Sky Survey (DSS) images of these galaxies are shown in Fig. 1 with the HST WFPC2 footprints superimposed. The field size of the red DSS-II images is $8^{\prime}$. The galaxies were usually centered on the WF3 chip. The WFPC2 images of the galaxies are presented in the upper panels of Fig. 1 in the Appendix, where both filters are combined.

For photometric measurements we used the HSTphot stellar photometry package developed by Dolphin (2000a). The package has been optimized for the undersampled conditions present in the WFPC2 to work in crowded fields. After removing cosmic rays, simultaneous photometry was performed on the F606W and F814W frames using multiphot, with corrections to the aperture with a radius of $0 .{ }^{\prime \prime} 5$. Charge-transfer efficiency (CTE) corrections and calibrations were then applied, which are based on the Dolphin (2000b) formulae, producing $V, I$ photometry for all stars detected in both images. Additionally, stars with a signal-to-noise ratio $S / N<3$, $|\chi|>2.0$, or $\mid$ sharpness $\mid>0.4$ in each exposure were eliminated from the final photometry list. The uncertainty of the photometric zero point is estimated to be within 0.05 (Dolphin 2000b).

\section{TRGB distances to five galaxies in the vicinity of IC 342}

The tip of the red giant branch (TRGB) method provides an efficient tool to measure galaxy distances. The TRGB distances agree with those given by the Cepheid period-luminosity relation to within 5\%. As shown by Lee et al. (1993), the TRGB is relatively independent of age and metallicity. In the $I$ band the TRGB for low-mass stars is found to be stable within $\sim 0^{\mathrm{m}} 1$ (Salaris \& Cassisi 1997; Udalski et al. 2001) for metallicities, $[\mathrm{Fe} / \mathrm{H}]$, encompassing the entire range from -2.1 to -0.7 dex found in metal-poor Galactic globular clusters. According to Da Costa \& Armandroff (1990), for metal-poor systems the TRGB is located at $M_{I}=-4$. 05 . Ferrarese et al. (2000) calibrated the zero point of the TRGB from galaxies with Cepheid distances and estimated $M_{I}=-4.06 \pm$ 0 . 07 (random) \pm 0.13 (systematic). A new TRGB calibration, $M_{I}=-4.04 \pm 0.12$, was made by Bellazzini et al. (2001) based on photometry and on the distance estimate from a detached eclipsing binary in the Galactic globular cluster $\omega$ Centauri. For this paper we use $M_{I}=-4$ m 05 . It should be mentioned that the distances to the Magellanic Clouds and to Milky Way globular clusters, which set the zero point of the extragalactic distance scale, are still a subject of discussions. For example, Udalski (2000) calibrated the TRGB method using red clump stars of the Magellanic Clouds and get $M_{I}$ value by 0.2 greater than adopted in our paper. In our work, we use the Da Costa \& Armandroff distance scale, which is consistent with the aforementioned Cepheid calibrations and which was also adopted in our preceding papers in this series 
Table 1. New distances to galaxies around IC 342.

\begin{tabular}{|c|c|c|c|c|c|c|c|c|c|}
\hline Name & $\begin{array}{c}\text { RA (J2000) Dec } \\
\text { h m s }{ }^{\prime \prime \prime \prime}\end{array}$ & $\begin{array}{l}a \\
b / a\end{array}$ & $\begin{array}{c}B_{\mathrm{t}} \\
A_{\mathrm{b}}\end{array}$ & $T$ & $\begin{array}{r}V_{\mathrm{h}} \\
V_{\mathrm{LG}}\end{array}$ & I(TRGB) & $(m-M)_{0}$ & $D_{\mathrm{MW}}$ & $\begin{array}{r}(V-I)_{-3.5} \\
{[\mathrm{Fe} / \mathrm{H}]}\end{array}$ \\
\hline CasdSph & $232631.8+504032$ & 2.5 & 13.65 & -3 & -307 & 20.83 & 24.50 & 0.79 & 1.39 \\
\hline KKH 96 & & 0.80 & 0.85 & & -5 & .05 & .10 & .04 & -1.55 \\
\hline \multirow[t]{2}{*}{ KK 35} & $034512.6+675150$ & 2.5 & 15.7 & 10 & -66 & 24.50 & 27.50 & 3.16 & - \\
\hline & & 0.68 & 2.34 & & 149 & .20 & .22 & .32 & \\
\hline \multirow[t]{2}{*}{ UGCA 86} & $035949.5+670731$ & 4.5 & 13.5 & 9 & 67 & $>24.50$ & $>26.7$ & $>2.2$ & - \\
\hline & & 0.69 & 3.82 & & 275 & & & & \\
\hline Cam A & $042515.6+724821$ & 3.7 & 14.85 & 10 & -47 & 24.33 & 27.97 & 3.93 & 1.38 \\
\hline KK 41 & & 0.57 & 0.95 & & 164 & .25 & .26 & .47 & -1.58 \\
\hline \multirow[t]{2}{*}{ N 1560} & $043249.9+715252$ & 9.8 & 11.90 & 8 & -36 & 24.01 & 27.69 & 3.45 & 1.43 \\
\hline & & 0.15 & 0.81 & & 171 & .21 & .23 & .36 & -1.42 \\
\hline Cam B & $045306.9+670557$ & 2.2 & 16.7 & 10 & 77 & 23.99 & 27.62 & 3.34 & 1.42 \\
\hline KK 44 & & 0.5 & 266 & .19 & .21 & .32 & -1.45 & & \\
\hline
\end{tabular}

(Karachentsev et al. 1999; 2000a,b, 2001a,b, 2002a-c, 2003a-c; Dolphin et al. 2001; Sarajedini et al. 2002; Makarova et al. 2002), thus ensuring that all of these distance measurements are on the same scale.

The lower left panels of Fig. 1 in the Appendix show I, $(V-I)$ color-magnitude diagrams (CMDs) for the six observed galaxies.

We determined the location of the TRGB using a Gaussiansmoothed $I$-band luminosity function (LF) for red stars with colors $V-I$ within \pm 0 . 5 of the mean $\langle V-I\rangle$ expected for red giant branch stars. Following Sakai et al. (1996), we applied a Sobel edge-detection filter. The position of the TRGB was identified with the peak in the filter response function. The resulting LFs and the Sobel-filtered LFs are shown in the lower right corners of Fig. 1 in the Appendix. The results are summarized in Table 1. There we list: (1) galaxy name; (2) equatorial coordinates of the galaxy center; (3) galaxy major axis diameter and axis ratio; (4) apparent integrated blue magnitude from the NASA Extragalactic Database (NED) and Galactic extinction in the $B$-band from Schlegel et al. (1998); (5) morphological type in de Vaucouleurs' notation; (6) heliocentric radial velocity and radial velocity with respect to the LG centroid (Karachentsev \& Makarov 1996); (7) position of the TRGB and its uncertainty as derived with the Sobel filter; (8) true distance modulus with its uncertainty, which takes into account the uncertainty in the TRGB, as well as uncertainties of the HST photometry zero point $(\sim 0.05)$, aperture corrections $(\sim 0.05)$, and crowding effects $\left(\sim 0{ }^{\mathrm{m}} 06\right)$ added quadratically; (for more details on the total budget of internal and external systematic errors for the TRGB method see Mendez et al. 2002); and (9) linear distance in $\mathrm{Mpc}$ and its uncertainty.

Given the distance moduli of the galaxies, we can estimate their mean metallicity from the mean color of the TRGB measured at the absolute magnitude $M_{I}=-3.5$, as recommended by Da Costa \& Armandroff (1990). Based on a Gaussian fit to the color distribution of the giant stars in a corresponding $I$-magnitude interval $(-3.5 \pm 0.3)$, we derived their mean colors, $(V-I)_{-3.5}$, which lie in the range of 1.38 to 1.43 after correction for Galactic reddening. Following the relation of Lee et al. (1993), this provides us with mean metallicities $-1.42 \mathrm{dex}>\langle[\mathrm{Fe} / \mathrm{H}]\rangle>-1.58 \mathrm{dex}$, listed in the last column of Table 1 . With the typical statistical scatter of the mean color $(\sim 0$ m 05$)$, and uncertainties of the HST photometry zero point we expect the uncertainty in metallicity to be about 0.3 dex. Therefore, within the measurement accuracy the metallicities of the galaxies satisfy the required limitation, $[\mathrm{Fe} / \mathrm{H}]<-0.7$ dex. Some individual properties of the galaxies are briefly discussed below.

Cas $d S p h=K K H$ 96. This dwarf spheroidal galaxy of very low surface brightness was discovered by Karachentsev \& Karachentseva (1999). Grebel \& Guhathakurta (1999) and Tikhonov \& Karachentsev (1999) detected its RGB population and determined the galaxy distance to be $760 \pm$ $70 \mathrm{kpc}$ and $708 \mathrm{kpc}$, respectively. According to Grebel \& Guhathakurta (1999), the central surface brightness of Cas dSph is $(24.2 \pm 0.2)^{\mathrm{m} / \square^{\prime \prime}}$ in the $V$ band. The galaxy radial velocity, $-307 \pm 2 \mathrm{~km} \mathrm{~s}^{-1}$, was measured by Guhathakurta et al. (2000; see Table 1 in Evans et al. 2000 for additional details). Our WFPC2 field covered only the central part of Cas dSph, nevertheless, we detected about 11000 stars seen in the galaxy in both filters. The CMD for them shows a sharp narrow branch of red giants. The TRGB position corresponds to $I($ TRGB $)=20.83 \pm 0.05$, which yields a distance of $0.79 \pm 0.04 \mathrm{Mpc}$, which is slightly higher than the previous TRGB estimates and agrees within the uncertainties with Grebel \& Guhathakurta's value. The distance is consistent with 
the distance of $\mathrm{M} 31,0.77 \pm 0.03 \mathrm{Mpc}$ derived from cepheids (Ferrarese et al. 2000), which confirms that Cas dSph is a peripheric companion to $M$ 31. For a complete listing of the presently known and presumed M 31 companions see Table 1 in Grebel et al. (2003). The deprojected distance of Cas dSph to $\mathrm{M} 31$ remains $\sim 220 \mathrm{kpc}$ as given in the preceding reference.

$K K 35$. This object was found as an isolated spot of very low surface brightness with a size of $2.5 \times 1.7$ situated at outer periphery of IC $342,16^{\prime}$ south of its center. It has been resolved into stars with CCD images obtained at the 6-m telescope (SAO RAS). Our surface photometry of KK 35 yielded a total magnitude $B_{\mathrm{T}}=15$. 7. Makarov \& Karachentsev (2003) determined its radial velocity, $-66 \pm 3 \mathrm{~km} \mathrm{~s}^{-1}$, from the $\mathrm{H}_{\alpha}$ line. The emission of H I with a velocity of $+105 \pm 2 \mathrm{~km} \mathrm{~s}^{-1}$, detected by Huchtmeier et al. (2003) in the direction to KK 35, apparently belongs to the Galactic hydrogen. Newton (1980) derived a detailed map of the H I distribution in IC 342. On this map the H I disc extends 40' away from the galaxy center, and in the KK 35 position an overdensity of H I with a radial velocity of about $-55 \mathrm{~km} \mathrm{~s}^{-1}$ is seen. These data show that KK 35 may be not an isolated dwarf galaxy, but a complex of young stars and gas on the outer disc of IC 342 itself. The CMD of KK 35 (Fig. 1 in the Appendix) shows the presence of a mixed population of blue and red stars. The TRGB is determined rather unreliably because of its close position to the photometric limit and the significant Galactic extinction. Adopting $I(\mathrm{TRGB})=24 \mathrm{~m} 50$, we obtain the distance to KK 35 to be $(3.16 \pm 0.32) \mathrm{Mpc}$ in agreement with the distance to IC $342,(3.28 \pm 0.26) \mathrm{Mpc}$, derived from cepheids.

UGCA 86. This Magellanic type irregular galaxy has an angular dimension of $4.6 \times 3.1$ and a total magnitude of $B_{\mathrm{T}}=13.5$. At its southern edge there is a bright region of active star formation, VII Zw9, which lies outside the WFPC2 view. Karachentsev et al. (1997) determined the galaxy distance to be $(2.65 \pm 0.53) \mathrm{Mpc}$ from the luminosity of the brightest blue and red stars. The extinction towards UGCA 86, $A_{B}=3$. 82 , was estimated based on the integrated galaxy color. It is slightly lower than the value $A_{B}=4$ m 06 from Schlegel et al. (1998). The central part of the galaxy imaged with WFPC2 contains about 9000 stars detected in both filters. The CMD for them is shown in Fig. 1 in the Appendix. Due to the significant color excess, $E(V-I)=1.21$, the tip of RGB is situated below the detection limit of our CCD images. Adopting $I($ TRGB $) \geq 24$. 5 , we obtain the lower limit of the distance to the galaxy, $D \geq 2.2 \mathrm{Mpc}$. Applying the Sobel edge-detection filter yields a peak at $I=24.14 \pm 0.18$, which is probably caused by the incompleteness of photometric data on red stars with colors $(V-I)>2.5 \mathrm{mag}$. Circumstantial evidence for a larger distance of UGCA 86 is also provided by its large positive velocity residual with regard to the main locus of the IC 342/Maffei group and of the LG (see Fig. 1 in Grebel 1997).

$\operatorname{Cam} A=K K 41$. This irregular galaxy of very low surface brightness with a size of $3.7 \times 2$.' 1 was found by Karachentsev (1994) and then included into the list of nearby galaxy candidates by Karachentseva \& Karachentsev (1998). Subarcsecond CCD images of Cam A in the $I$ band have been obtained at the 2.6-m Nordic telescope by Karachentsev et al. (1999), who estimated the galaxy distance to be $1.9 \mathrm{Mpc}$. Gallart et al. (1999) made deeper CCD imaging of Cam A in the V,I bands and noted that the galaxy may be situated at a much larger distance. According to the latest observations by Makarov \& Karachentsev (2003) and Huchtmeier et al. (2003), Cam A has been detected in the $\mathrm{H}_{\alpha}$ line and in the $\mathrm{HI}$ with a radial velocity of $(-49 \pm 3) \mathrm{km} \mathrm{s}^{-1}$ and $(-47 \pm 1) \mathrm{km} \mathrm{s}^{-1}$, respectively. The CMD of the central part of Cam A (Fig. 1 in the Appendix) contains about 3800 blue and red stars, particularly, RGB stars. The TRGB position corresponds to $I(\mathrm{TRGB})=24.33 \pm 0 \mathrm{~m} \cdot 25$, yielding a distance $(3.93 \pm 0.47) \mathrm{Mpc}$.

$N G C$ 1560. This edge-on Sdm galaxy was resolved into stars for the first time by Karachentsev et al. (1991), who determined its distance to be $(2.98 \pm 0.56) \mathrm{Mpc}$ based on the luminosity of the brightest stars. Being of $9.8 \times 1.5$ in size, NGC 1569 extends far beyond the WFPC2 field of view. In its central part our HST photometry reveals about 16000 stars. Their CM diagram in Fig. 1 in the Appendix shows the presence of a mixed population of blue and red stars, in particular, RGB and AGB stars. Applying the Sobel filter yields $I(\mathrm{TRGB})=24.01 \pm 0.21$ and a corresponding distance of $(3.45 \pm 0.36) \mathrm{Mpc}$. It appears from Table 1 that NGC 1560 and Cam A are separated by a projected distance of 1.1 or $\sim 65 \mathrm{kpc}$, and their radial velocity difference is only $7 \mathrm{~km} \mathrm{~s}^{-1}$. The derived distances of NGC 1560 and Cam A are consistent with the idea that they form a relatively isolated pair inside the IC 342/Maffei group.

Cam $B=K K$ 44. This irregular galaxy of low surface brightness with an angular dimension of $2.2 \times 1.1$ was found by Karachentseva \& Karachentsev (1998) and then detected in the H I line by Huchtmeier et al. (1997, 2003). Our HST photometry of Cam B finds about 1600 stars belonging mostly to RGB (see Fig. 1 in the Appendix). Based on the TRGB position, (23. $99 \pm 0.19)$, we derived a galaxy distance of (3.34 \pm $0.32) \mathrm{Mpc}$, which shows Cam B to be an IC 342 group member.

\section{On the structure and kinematics of the IC 342/Maffei complex}

To study the 3-D structure of the IC 342/Maffei group, we collected the most complete sample of data on all known nearby galaxies situated in this region of the sky using our own data and data from the literature. Table 2 presents the following characteristics of the 26 galaxies in our sample: (1) galaxy name; (2) equatorial (epoch 1950.0) coordinates; (3) angular separation $\theta$ from IC 342 in degrees; (4) morphological type; (5) heliocentric radial velocity in $\mathrm{km} \mathrm{s}^{-1}$, (6) radial velocity in the LG rest frame (Karachentsev \& Makarov 1996); (7) $\mathrm{HI}$ line width (in $\mathrm{km} \mathrm{s}^{-1}$ ) at the $50 \%$ level of the maximum from LEDA (Paturel et al. 1996); (8) apparent integrated magnitude from NED or Buta \& McCall (1999); for some galaxies the blue total magnitudes were estimated from the known $V$ and $I$ magnitudes based on the mean color-color relation, $(B-V)_{0}=0.85(V-I)_{0}-0.20$, which is valid for latetype galaxies (Makarova 1999); (9) Galactic extinction in the $B$-band from Schlegel et al. (1998); the values indicated with an asterisk were estimated from the individual color excess of the galaxies; (10) distance to the galaxy in Mpc and method used 
Table 2. List of members and probable members of the binary group IC 342/Maffei.

\begin{tabular}{|c|c|c|c|c|c|c|c|c|c|c|c|}
\hline Name & $\begin{array}{c}\text { RA(J2000.0)Dec } \\
\text { h m s }, \prime \prime\end{array}$ & $\begin{array}{l}\theta \\
\circ\end{array}$ & $T$ & $\begin{array}{r}V_{\mathrm{h}} \\
\mathrm{km} \mathrm{s}^{-1}\end{array}$ & $\begin{array}{r}V_{\mathrm{LG}} \\
\mathrm{km} \mathrm{s}^{-1}\end{array}$ & $\begin{array}{r}W \\
\mathrm{~km} \mathrm{~s}^{-1}\end{array}$ & $\begin{array}{r}B_{\mathrm{t}} \\
\mathrm{mag}\end{array}$ & $\begin{array}{l}A_{\mathrm{b}} \\
\mathrm{mag}\end{array}$ & $\begin{array}{l}\text { Distance } \\
\mathrm{Mpc}\end{array}$ & $\begin{array}{l}M_{B} \\
\text { mag }\end{array}$ & Ref. \\
\hline KKH 5 & $010732.5+512625$ & 25.28 & 10 & 61 & 326 & 37 & 17.1 & 1.19 & $4.26 \mathrm{rgb}$ & -12.2 & (1) \\
\hline KKH 6 & $013451.6+520530$ & 22.46 & 10 & 17 & 270 & 19 & 17.0 & 1.52 & $3.72 \mathrm{H} 72$ & -12.4 & \\
\hline Cas 1 & $020607.9+690036$ & 9.29 & 10 & 35 & 283 & 50 & 15.31 & 4.40 & $3.3 \mathrm{mem}$ & -16.7 & \\
\hline KKH 11 & $022435.0+560042$ & 15.30 & 10 & 75 & 308 & 92 & 16.2 & 2.13 & $3.0 \mathrm{mem}$ & -13.3 & \\
\hline KKH 12 & $022727.0+572916$ & 13.78 & 10 & 70 & 303 & 52 & 17.8 & 3.44 & $3.0 \mathrm{mem}$ & -13.0 & \\
\hline MB 1 & $023535.6+592247$ & 11.66 & 10 & 190 & 421 & 50 & 16.8 & 4.22 & $3.0 \mathrm{mem}$ & -14.8 & \\
\hline Maffei 1 & $023635.5+593918$ & 11.37 & -5 & 66 & 297 & $187^{*}$ & 13.47 & $6.08 *$ & 3.0 DnS & -20.0 & (2) \\
\hline Maffei 2 & $024154.5+593611$ & 11.04 & 4 & -17 & 212 & 305 & 14.77 & $7.19 *$ & $2.8 \mathrm{tf}$ & -19.7 & (3) \\
\hline Dw 2 & $025408.5+590019$ & 10.77 & 10 & 94 & 316 & 90 & 17.97 & 5.13 & $3.0 \mathrm{mem}$ & -14.5 & \\
\hline MB 3 & $025543.6+585142$ & 10.81 & 10 & 59 & 280 & 15 & 19.38 & 5.64 & $3.0 \mathrm{mem}$ & -13.7 & \\
\hline Dw 1 & $025656.1+585442$ & 10.70 & 5 & 112 & 333 & 187 & 15.01 & 6.34 & $2.8 \mathrm{tf}$ & -18.6 & (3) \\
\hline KK 35 & $034512.6+675150$ & 0.27 & 10 & -66 & 149 & 34 & 15.7 & 2.34 & $3.16 \mathrm{rgb}$ & -14.2 & (3) \\
\hline IC 342 & $034649.7+680545$ & 0.00 & 5 & 31 & 245 & 151 & 9.37 & $2.62 *$ & 3.28 cep & -20.8 & (4) \\
\hline UA 86 & $035949.5+670731$ & 1.59 & 8 & 67 & 275 & 83 & 13.5 & $3.82 *$ & $2.65 \mathrm{bs}$ & -17.4 & (5) \\
\hline Cam A & $042515.6+724821$ & 5.69 & 10 & -47 & 164 & 44 & 14.85 & 0.95 & $3.93 \mathrm{rgb}$ & & (3) \\
\hline N 1569 & $043049.1+645053$ & 5.55 & 9 & -104 & 88 & 74 & 11.30 & 2.89 & $1.95 \mathrm{rgb}$ & & (6) \\
\hline N 1560 & $043249.9+715252$ & 5.44 & 8 & -36 & 171 & 125 & 11.90 & 0.81 & $3.45 \mathrm{rgb}$ & -16.6 & (3) \\
\hline UA 92 & $043200.3+633650$ & 6.54 & 10 & -99 & 89 & 77 & 15.22 & 3.42 & $1.8 \mathrm{bs}$ & -14.5 & (5) \\
\hline Cam B & $045306.9+670557$ & 6.47 & 10 & 77 & 266 & 20 & 16.7 & 0.94 & $3.34 \mathrm{rgb}$ & -11.8 & (3) \\
\hline UA 105 & $051415.1+623451$ & 10.80 & 9 & 111 & 279 & 118 & 12.14 & 1.35 & $3.15 \mathrm{rgb}$ & -16.7 & (7) \\
\hline ККН 34 & $055941.2+732539$ & 11.97 & 10 & 110 & 299 & 24 & 17.1 & 1.08 & $4.61 \mathrm{rgb}$ & -12.3 & (1) \\
\hline KKH 37 & $064745.8+800726$ & 16.50 & 10 & 11 & 214 & 20 & 16.4 & 0.33 & $2.97 \mathrm{H} 72$ & -11.3 & \\
\hline N 2366 & $072854.6+691257$ & 19.76 & 10 & 100 & 254 & 96 & 11.43 & 0.16 & $3.19 \mathrm{rgb}$ & -16.2 & (8) \\
\hline DDO 44 & $073411.3+665310$ & 21.38 & -3 & - & - & - & 15.64 & 0.18 & $3.19 \mathrm{rgb}$ & -12.1 & (8) \\
\hline N 2403 & $073654.4+653558$ & 22.36 & 6 & 131 & 268 & 231 & 8.82 & 0.18 & 3.30 cep & -19.0 & (8) \\
\hline CasDSph & $232631.8+504032$ & 35.30 & -3 & -307 & -5 & - & 13.65 & 0.85 & $0.79 \mathrm{rgb}$ & -11.7 & (3) \\
\hline
\end{tabular}

(1) Karachentsev et al. (2003a), (2) Fingerhut et al. (2003), (3) present paper, (4) Saha et al. (2002), (5) Karachentsev et al. (1997), (6) Makarova \& Karachentsev (2003), (7) Karachentsev et al. (2002a), (8) Karachentsev et al. (2002b).

for distance measurement ("Cep" - from Cepheids, "RGB" from the TRGB, "bs" - from the luminosity of brightest stars, "mem" - from assumed subgroup membership, "H72" - from the Hubble relation with $H_{0}=72 \mathrm{~km} \mathrm{~s}^{-1} \mathrm{Mpc}^{-1}$ ); here the distances to two spiral galaxies, Maffei 2 and Dwingeloo 2, were estimated by us from the Tully-Fisher relation, $M_{B}=$ $-7.0 \log \left(W_{\mathrm{c}}\right)-1.8$, where $W_{\mathrm{c}}$ is the $\mathrm{H}$ I line width corrected for the galaxy inclination; (11) absolute magnitude of the galaxy. The last column gives the distance data reference.

The distribution of the 26 galaxies from Table 2 is presented in Fig. 2 in Galactic coordinates. The radial velocities of the galaxies with respect to the LG centroid are indicated by numbers. The galaxies prove to concentrate around two centers, IC 342 and Maffei 1. However, the empty zone seen between them may also be caused by strong Galactic extinction. Referring to Table 2, we recognize the luminosity of the giant spiral galaxy IC 342 to be more than twice as high as that of all other galaxies in this complex. Therefore, we consider IC 342 as the dynamical center of the whole group.
The Hubble diagram showing velocity versus distance for the 26 galaxies is given in Fig. 3. Galaxies with accurate distance estimates ("Cep", "RGB") are shown by filled circles, while galaxies with poorly determined distances are indicated by crosses, and the brightest galaxies, IC 342 and Maffei 1, are shown as squares. The solid line corresponds to the Hubble parameter $H=72 \mathrm{~km} \mathrm{~s}^{-1} \mathrm{Mpc}^{-1}$. At small distances the line deviates from a straight line because of the decelerating gravitational action of the Local Group, the total mass of which is adopted to be $M_{\mathrm{LG}}=1.3 \times 10^{12} M_{\odot}$ (Karachentsev et al. 2002a). Note that with respect to the Hubble regression, IC 342 has a low peculiar velocity, $+27 \mathrm{~km} \mathrm{~s}^{-1}$.

Based on the data in Figs. 2 and 3, we can describe the structure of the galaxy complex in the following way. Apart from the galaxies apparently belonging to the foreground (Cas dSph, NGC 1569, UGCA 92), background (KKH 5, KKH 6, KKH 34 and, probably, KKH 37), and the M 81 group outskirts (NGC 2366, DDO 44, NGC 2403), the remaining 16 galaxies seem to be members of the binary group 


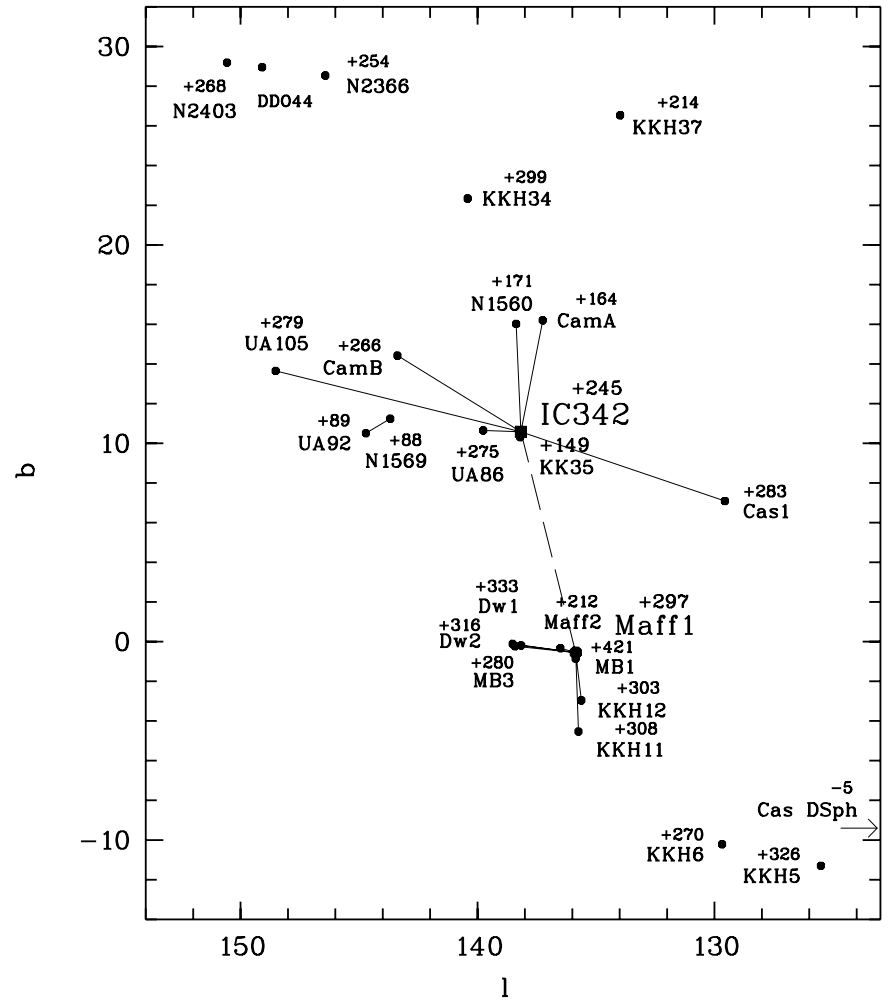

Fig. 2. Sky distribution of nearby galaxies in the direction towards IC 342 and Maffei 1. Probable companions to IC 342 and Maffei 1 are connected to the principal galaxies with straight lines. Radial velocities of the galaxies relative to the Local Group centroid are indicated by numbers.

IC 342/Maffei1. Among them the 7 dwarf galaxies, KK 35, UGCA 86, NGC 1560, Cam A, Cam B, Cas 1, and UGCA 105, are probable companions of IC 342. The seven galaxies Maffei 2, MB 1, MB 3, Dw 1, Dw 2, KKH 12, and KKH 11 are associated with Maffei 1. In Fig. 2 these companions are connected to the principal galaxies with straight lines.

It should be mentioned that we also obtained the WFPC2 images of seven more objects in the IC 342/Maffei region. Two of them, MB 2 and Cam C = KK 26, turn out to be Galactic cirrus and a Galactic H II region, respectively. The other five galaxies, Cas 1, Maffei 1, Maffei 2, MB 1, and Dw 2 are too strongly obscured, which does not allow us to detect their TRGB.

\section{Mass estimates of the groups}

The total mass of each group can be estimated from the virial balance of kinetic and potential energies (Limber \& Mathews 1960),

$M_{\mathrm{vir}}=3 \pi N(N-1)^{-1} G^{-1} \sigma_{\mathrm{v}}^{2} R_{\mathrm{H}}$,

where $N$ is the number of galaxies in the group, $\sigma_{\mathrm{v}}^{2}$ is the radial velocity dispersion, $R_{\mathrm{H}}$ is the mean projected harmonic radius, and $G$ is the gravitational constant. This approach assumes that the characteristic crossing time of the group, $T_{\text {cross }}=\left\langle R_{\mathrm{p}}\right\rangle / \sigma_{\mathrm{v}}$, is low in comparison to the age of the Universe (here $\left\langle R_{\mathrm{p}}\right\rangle$ means the average projected radius of the group).

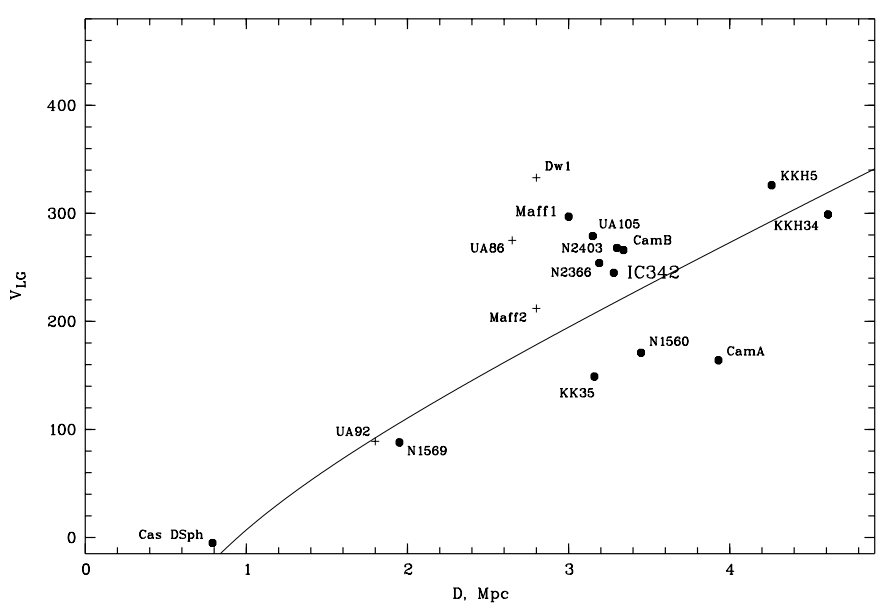

Fig. 3. Radial velocity - distance relation for 17 nearby galaxies around IC 342. The galaxies with accurate distance estimates are shown as filled circles, and galaxies with less reliable distances are shown as crosses. The solid line corresponds to the Hubble relation with $H_{0}=72 \mathrm{~km} \mathrm{~s}^{-1} \mathrm{Mpc}^{-1}$, curved at small distances assuming a decelerating gravitational action of the Local Group with a total mass of $1.3 \times 10^{12} M_{\odot}$.

Table 3. Properties of the groups around IC 342 and Maffei 1.

\begin{tabular}{lcc}
\hline \hline Parameter & IC 342 & Maffei 1 \\
\hline$N$ & 8 & 8 \\
$<V>, \mathrm{km} \mathrm{s}^{-1}$ & 229 & 309 \\
$<D>, \mathrm{Mpc}$ & 3.28 & 3.0 \\
$<R_{\mathrm{p}}>, \mathrm{kpc}$ & 322 & 112 \\
$R_{\mathrm{H}}, \mathrm{kpc}$ & 78 & 56 \\
$\sigma_{\mathrm{v}}, \mathrm{km} \mathrm{s}^{-1}$ & 60 & 59 \\
$L_{B}, 10^{10} L_{\odot}$ & 3.43 & 2.97 \\
$M_{\mathrm{vir}}, 10^{10} M_{\odot}$ & 70 & 49 \\
$M_{\text {orb }}, 10^{10} M_{\odot}$ & 95 & 14 \\
$M_{\text {vir }} / L_{B}, M_{\odot} / L_{\odot}$ & 20.4 & 16.5 \\
$M_{\text {orb }} / L_{B}, M_{\odot} / L_{\odot}$ & 27.7 & 4.7 \\
$T_{\text {cross }}, \mathrm{Gyr}$ & 5.4 & 1.9 \\
\hline & &
\end{tabular}

Another way to estimate the total mass of a group was proposed by Bahcall \& Tremaine (1981). Assuming the motions of dwarf galaxies around the main group member to be closed Keplerian motions with orbit eccentricity $e$, in the case of random orientation of galaxy orbits we obtain

$M_{\text {orb }}=(32 / 3 \pi) G^{-1}\left(1-2 e^{2} / 3\right)^{-1}\left\langle R_{\mathrm{p}} \cdot \Delta V_{\mathrm{r}}^{2}\right\rangle$,

where $R_{\mathrm{p}}$ and $\Delta V$ are the projected distance and radial velocity of the companion with respect to the main group member. The basic dynamical parameters of the groups around IC 342 and Maffei 1 are presented in Table 3. In the case of orbital mass estimates the mean eccentricity $e=0.7$ is adopted. As seen from these data, the crossing time of both groups, 5.4 Gyr and $1.9 \mathrm{Gyr}$, is shorter than the time of cosmic expansion, $1 / H_{0}$. The virial/orbital mass-to-luminosity ratios of the groups lie in the range 5 to $28 M_{\odot} / L_{\odot}$. 


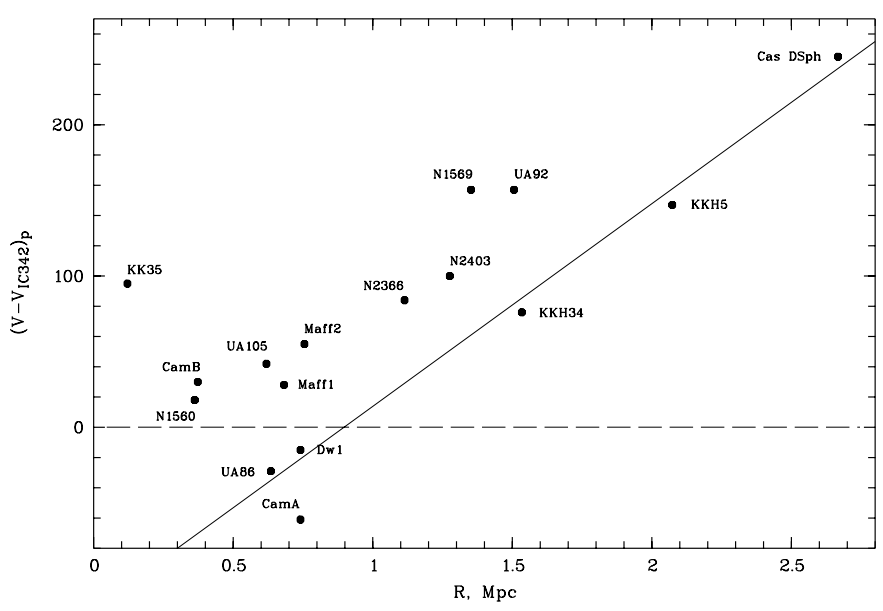

Fig. 4. The distribution of the radial velocity difference and of the space distance of nearby galaxies with respect to IC 342 . These data yield a radius of the zero-velocity surface of $R_{0}=0.9 \mathrm{Mpc}$.

According to Lynden-Bell (1981) and Sandage (1986), in the expanding universe any group dense enough with a total mass $M_{0}$ may be characterized by a "zero-velocity surface", which separates the group from the Hubble flow. In the case of spherical symmetry, the radius of this surface, $R_{0}$, is expressed via the total mass of the group and the Hubble constant, $H_{0}$, by a simple relation

$$
M_{0}=\left(\pi^{2} / 8 G\right) H_{0}^{2} R_{0}^{3} .
$$

For estimating $R_{0}$, we calculated the spatial separation from IC 342 for any galaxy with a distance $D$ and a radial velocity $V$,

$R^{2}=D^{2}+D_{\text {IC } 342}^{2}-2 D \cdot D_{\text {IC } 342} \cos \theta$,

and its projected radial velocity with respect to IC 342 ,

$\left(V-V_{\text {IC } 342}\right)_{\mathrm{p}}=V \cos \lambda-V_{\mathrm{IC} 342} \cos (\theta+\lambda)$,

where $\tan \lambda=D_{\text {IC } 342} \sin \theta /\left(D-D_{\text {IC } 342} \cos \theta\right)$. Here we assumed that the peculiar velocities of the galaxies are low in comparison with the velocities of the regular Hubble flow. The estimated values $\left(V-V_{\text {IC } 342}\right)_{\mathrm{p}}$ and $R$ for 16 galaxies around IC 342 are presented in Fig. 4. It appears that all galaxies outside $R=0.9 \mathrm{Mpc}$ move away from IC 342. Based on these (still incomplete) data, we can conclude that the radius of the zerovelocity surface for the IC 342 group is $R_{0}=0.9 \pm 0.1 \mathrm{Mpc}$. According to Eq. (3) this corresponds to a total mass of $M_{\mathrm{T}}=$ $(107 \pm 33) \times 10^{10} M_{\odot}$ or $M_{\mathrm{T}} / L_{B}=(17 \pm 5) M_{\odot} / L_{\odot}$, which is consistent with the virial/orbital mass estimates derived above.

Note, however, that more than half of the IC 342/Maffei group galaxies do not yet have accurate distance measurements. In particular, some authors presented arguments in favour of a long distance scale of $\sim(4-5)$ Mpc to Maffei 1 (Luppino \& Tonry 1993; Davidge \& van den Bergh 2002) and Dw 1 (Ivanov et al. 1999). So far, we have no reliable observational data on distances to Maffei 1 companions to decide whether the IC 342 group and the Maffei group are moving away from each other or merging.

\section{Concluding remarks}

New accurate measurements of distances to galaxies around IC 342 led to a better understanding of the structure and kinematics of this nearest complex of galaxies strongly obscured by Galactic dust clouds. The IC 342 group itself contains 8 probable members, which have a mean distance of $(3.28 \pm 0.15) \mathrm{Mpc}$ and a mean radial velocity of $(229 \pm 23) \mathrm{km} \mathrm{s}^{-1}$. The IC 342 group is characterized by a projected radius of $322 \mathrm{kpc}$, a radial velocity dispersion of $60 \mathrm{~km} \mathrm{~s}^{-1}$, and a total blue luminosity $3.43 \times 10^{10} L_{\odot}$. We derived the virial mass-to-luminosity ratio of the group to be in the range of [20-28] $M_{\odot} / L_{\odot}$. The peculiar radial velocity of IC 342 with respect to the Hubble flow (with $H_{0}=72 \mathrm{~km} \mathrm{~s}^{-1} \mathrm{Mpc}^{-1}$ ) is only $+27 \mathrm{~km} \mathrm{~s}^{-1}$.

The galaxy group around Maffei 1 has so far a less reliable distance of $\sim 3.0 \mathrm{Mpc}$ (Fingerhut et al. 2003) and a mean radial velocity of $(309 \pm 22) \mathrm{km} \mathrm{s}^{-1}$. The Maffei group contains 8 probable members as well, but most of them have been discovered only in the last few years. It seems likely that the total population of both groups is underestimated because of the strong Galactic extinction. The Maffei group is characterized by a projected radius of $112 \mathrm{kpc}$, a radial velocity dispersion of $59 \mathrm{~km} \mathrm{~s}^{-1}$, a total blue luminosity of $2.97 \times 10^{10} L_{\odot}$, and a virial mass-to-luminosity ratio in the range of [5-17] $M_{\odot} / L_{\odot}$. Therefore, both groups have rather low virial mass-to-luminosity ratio estimates. The sum of the virial $\left(119 \times 10^{10} M_{\odot}\right)$ and orbital $\left(109 \times 10^{10} M_{\odot}\right)$ mass estimates well agree with the total mass of the IC 342/Maffei complex $(107 \pm 33) \times 10^{10} M_{\odot}$, derived from its radius of the "zero velocity surface", $R_{0}=(0.9 \pm 0.1) \mathrm{Mpc}$, which separates the complex from the general Hubble flow. The centers of both the groups are separated by a projected distance of $\sim 700 \mathrm{kpc}$. At present, it is unclear whether the group centers are approaching each other (like M 31 and the Milky Way), or are moving away from each other (like in the Cen A and M 83 groups; see Karachentsev et al. 2002c).

Acknowledgements. Support for this work was provided by NASA through grant GO-08601.01-A from the Space Telescope Science Institute, which is operated by the Association of Universities for Research in Astronomy, Inc., under NASA contract NAS5-26555. This work was partially supported by RFBR grant 01-02-16001 and DFG-RFBR grant 02-02-04012.

The Digitized Sky Surveys were produced at the Space Telescope Science Institute under U.S. Government grant NAG W-2166. The images of these surveys are based on photographic data obtained using the Oschin Schmidt Telescope on the Palomar Mountain and the UK Schmidt Telescope. The plates were converted into the present compressed digital form with permission of these institutions.

This project made use of the NASA/IPAC Extragalactic Database (NED), which is operated by the Jet Propulsion Laboratory, Caltech, under contract with the National Aeronautics and Space Administration.

\section{References}

Bahcall, J. N., \& Tremaine, S. 1981, ApJ, 244, 805

Bellazzini, M., Ferraro, F. R., \& Pancino, E. 2001, ApJ, 556, 635 
Buta, R. J., \& McCall, M. L. 1999, ApJS, 124, 33

Da Costa, G. S., \& Armandroff, T. E. 1990, AJ, 100, 162

Davidge, T. J., \& van den Bergh, S. 2002, AJ, 124, 2012

Dolphin, A. E. 2000a, PASP, 112, 1383

Dolphin, A. E. 2000b, PASP, 112, 1397

Dolphin, A. E., Makarova, L. Karachentsev, I. D., et al. 2001, MNRAS, 324, 249

Evans, N. W., Wilkinson, M. I., Guhathakurta, P., et al. 2000, ApJ, 540, L9

Ferrarese, L., Mould, J. R., Kennicut, R. C. Jr., et al. 2000, ApJ, 529, 745

Fingerhut, R. L., McCall, M. L., De Robertis, M., et al. 2003, ApJ, 587,672

Gallart, C., Martinez-Delgado, D., Aparicio, A., \& Freedman, W. L. 1999, in The Stellar Content of the Local Group, ed. P. Whitelock, \& R. Cannon (San Francisco: ASP), IAU Symp., 192, 284

Grebel, E. K. 1997, RvMA, 10, 29

Grebel, E. K., \& Guhathakurta, P. 1999, ApJ, 511, 101

Grebel, E. K., Seitzer, P., Dolphin, A. E., et al. 2000, in Stars, Gas and Dust in Galaxies: Exploring the Links, ed. D. Alloin, K. Olsen, \& C. Galaz (San Francisco: ASP), ASP Conf. Ser., 221, 147

Grebel, E. K., Gallagher, J. S., \& Harbeck, D. 2003, AJ, 125, 1926

Guhathakurta, P., Reitzel, D. B., \& Grebel, E. K. 2000, SPIE, 4005, 168

Huchtmeier, W. K., Karachentsev, I. D., \& Karachentseva, V. E. 2003 , A\&A, 401, 483

Huchtmeier, W. K., Karachentsev, I. D., \& Karachentseva, V. E. 2000, ed. M. J. Valtonen, \& C. Flynn (San Francisco: ASP), ASP Conf. Ser., 209, 158

Huchtmeier, W. K., Lercher, G., Seeberger, R., et al. 1995, A\&A, 293, L33

Huchtmeier, W. K., \& van Driel, W. 1996, A\&A, 305, L25

Huchtmeier, W. K., Karachentsev, I. D., \& Karachentseva, V. E. 1997, A\&A, 322, 375

Ivanov, V. D., Alonso-Herrero, A., Reike, M. J., \& McCarthy, D. 1999, AJ, 118, 826

Karachentsev, I. D. 1994, A\&Ap Trans., 6, 3

Karachentsev, I., \& Makarov, D. 1996, AJ, 111, 535

Karachentsev, I. D., \& Karachentseva, V. E. 1999, A\&A, 341, 355

Karachentsev, I. D., Makarova, L. N., \& Andersen, M. I. 1999, MNRAS, 307, L37

Karachentsev, I. D., Tikhonov, N. A., Georgiev, Z. B., et al. 1991, A\&AS, 91, 503

Karachentsev, I. D., Karachentseva, V. E., \& Huchtmeier, W. K. 2001, A\&A, 366, 428

Karachentsev, I. D., Drozdovsky, I., Kajsin, S., et al. 1997, A\&AS, 124,559

Karachentsev, I. D., Sharina, M. E., Grebel, E. K., et al. 1999, A\&A, 352,399

Karachentsev, I. D., Sharina, M. E., Grebel, E. K., et al. 2000a, ApJ, 542,128

Karachentsev, I. D., Karachentseva, V. E., Dolphin, A. E., et al. 2000b, A\&A, 363, 117
Karachentsev, I. D., Sharina, M. E., Dolphin, A. E., et al. 2001a, A\&A, 375,359

Karachentsev, I. D., Sharina, M. E., Dolphin, A. E., et al. 2001b, A\&A, 379,407

Karachentsev, I. D., Sharina, M. E., Makarov, D. I., et al. 2002a, A\&A, 389,812

Karachentsev, I. D., Dolphin, A. E., Geisler, D., et al. 2002b, A\&A, 383,125

Karachentsev, I. D., Sharina, M. E., Dolphin, A. E., et al. 2002c, A\&A, 385,21

Karachentsev, I. D., Makarov, D. I., Sharina, M. E., et al. 2003a, A\&A, 398, 479

Karachentsev, I. D., Sharina, M. E., Dolphin, A. E., et al. 2003, A\&A, 398, 467

Karachentsev, I. D., Grebel, E. K., Sharina, M. E., et al. 2003, A\&A, 404, 93

Karachentseva, V. E., \& Karachentsev, I. D. 1998, A\&AS, 127, 409

Kraan-Korteweg, R. C., Loan, A. J., Burton, W. B., et al. 1994, Nature, 372,77

Krishmer, M., Tully, R. B., \& Gioia, I. M. 1995, AJ, 110, 1584

Lee, M. G., Freedman, W. L., \& Madore, B. F. 1993, ApJ, 417, 553

Limber, D. N., \& Mathews, W. G. 1960, ApJ, 132, 286

Luppino, G. A., \& Tonry, J. L. 1993, ApJ, 410, 81

Lynden-Bell, D. 1981, Observatory, 101, 111

Makarov, D. I., \& Karachentsev, I. D. 2003, A\&A, accepted

Makarova, L. N. 1999, A\&AS, 139, 491

Makarova, L. N., \& Karachentsev, I. D. 2003, Astrofizika, 46, 181

Makarova, L. N., Grebel, E. K., Karachentsev, I. D., et al. 2002, A\&A, 396, 473

McCall, M. L., \& Buta, R. J. 1995, 109, 2460

Mendez, B., Davis, M., Moustakas, J., et al. 2002, AJ, 124, 213

Newton, K. 1980, MNRAS, 191, 169

Paturel, G., Bottinelli, L., Di Nella, H., et al. 1996, Catalogue of Principal Galaxies (LEDA), Saint-Genis Laval, Observatoire de Lyon

Peebles, P. J. E. 1994, ApJ, 429, 43

Saha, A., Claver, J., \& Hoessel, J. G. 2002, AJ, 124, 839

Sakai, S., Madore, B. F., \& Freedman, W. L. 1996, ApJ, 461, 713

Salaris, M., \& Cassisi, S. 1997, MNRAS, 289, 406

Sandage, A. 1986, ApJ, 307, 1

Sarajedini, A., Grebel, E. K., Dolphin, A. E., et al. 2002, ApJ, 567, 915

Schlegel, D. J., Finkbeiner, D. P., \& Davis, M. 1998, ApJ, 500, 525

Seitzer, P., Grebel, E. K., Dolphin, A. E., et al. 1999, AAS, 195, 0801

Tikhonov, N. A., \& Karachentsev, I. D. 1999, Lett. Astron. Zh., 25, 391

Udalski, A. 2000, Acta Astron., 50, 279

Udalski, A., Wyrzykowski, L., Pietrzynski, G., et al. 2001, Acta Astron., 51, 221

Valtonen, M. J., Byrd, G. G., McCall, M. L., \& Innanen, K. A. 1993, AJ, 105, 886

Zheng, J. Q., Valtonen, M. J., \& Byrd, G. G. 1991, A\&A, 247, 20 


\section{Online Material}



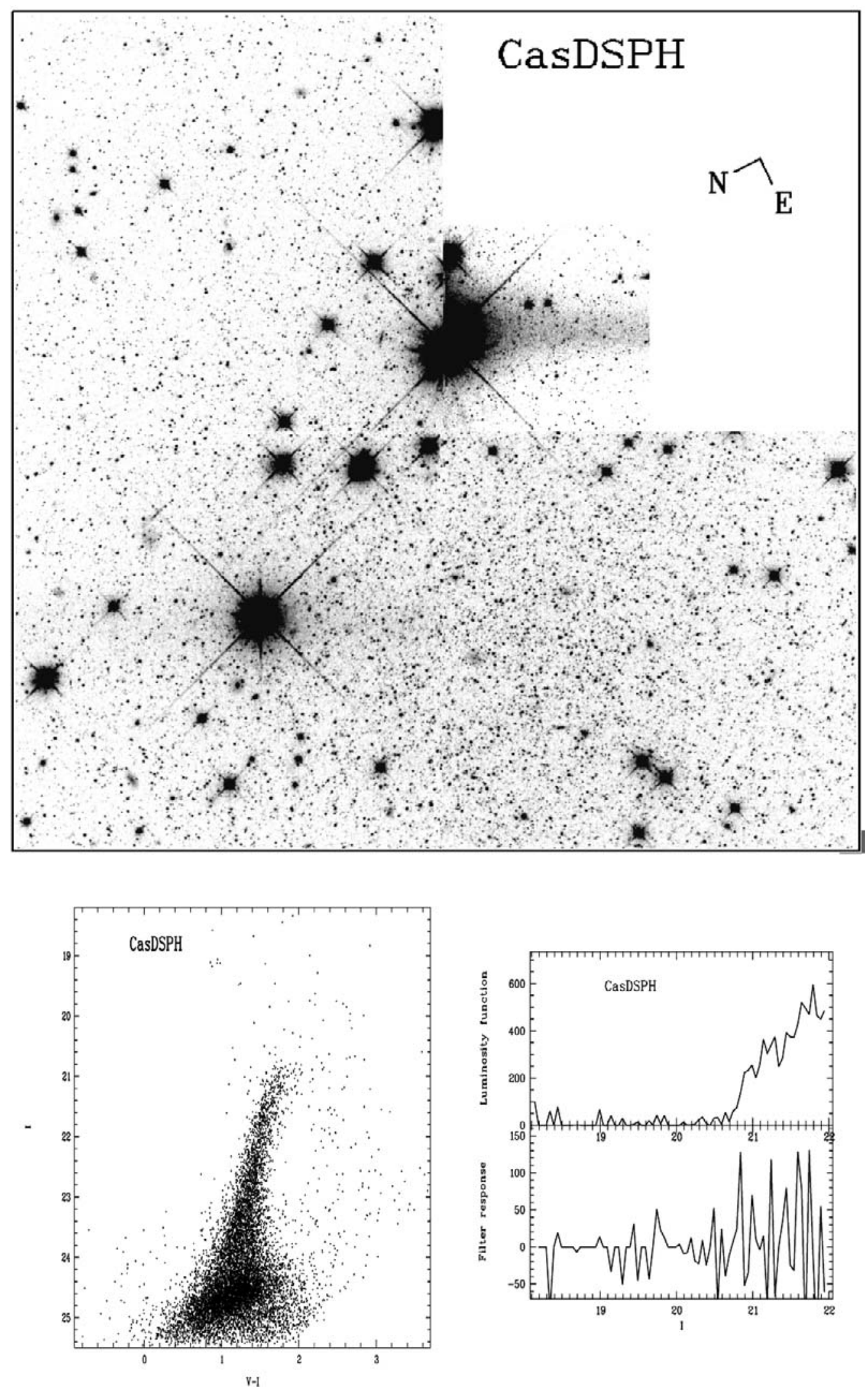

Fig. A.1. Top: WFPC2 images of six galaxies: Cas dSph, KK 35, UGCA 86, Cam A, NGC 1560, and Cam B produced by combining the two $600 \mathrm{~s}$ exposures obtained through the F606W and F814W filters. The arrows point to the North and the East. Bottom left: the color-magnitude diagrams from the WFPC2 data for the six galaxies around IC 342. Bottom right: the Gaussian-smoothed $I$-band luminosity function restricted to red stars (top), and the output of an edge-detection filter applied to the luminosity function for the six galaxies. 

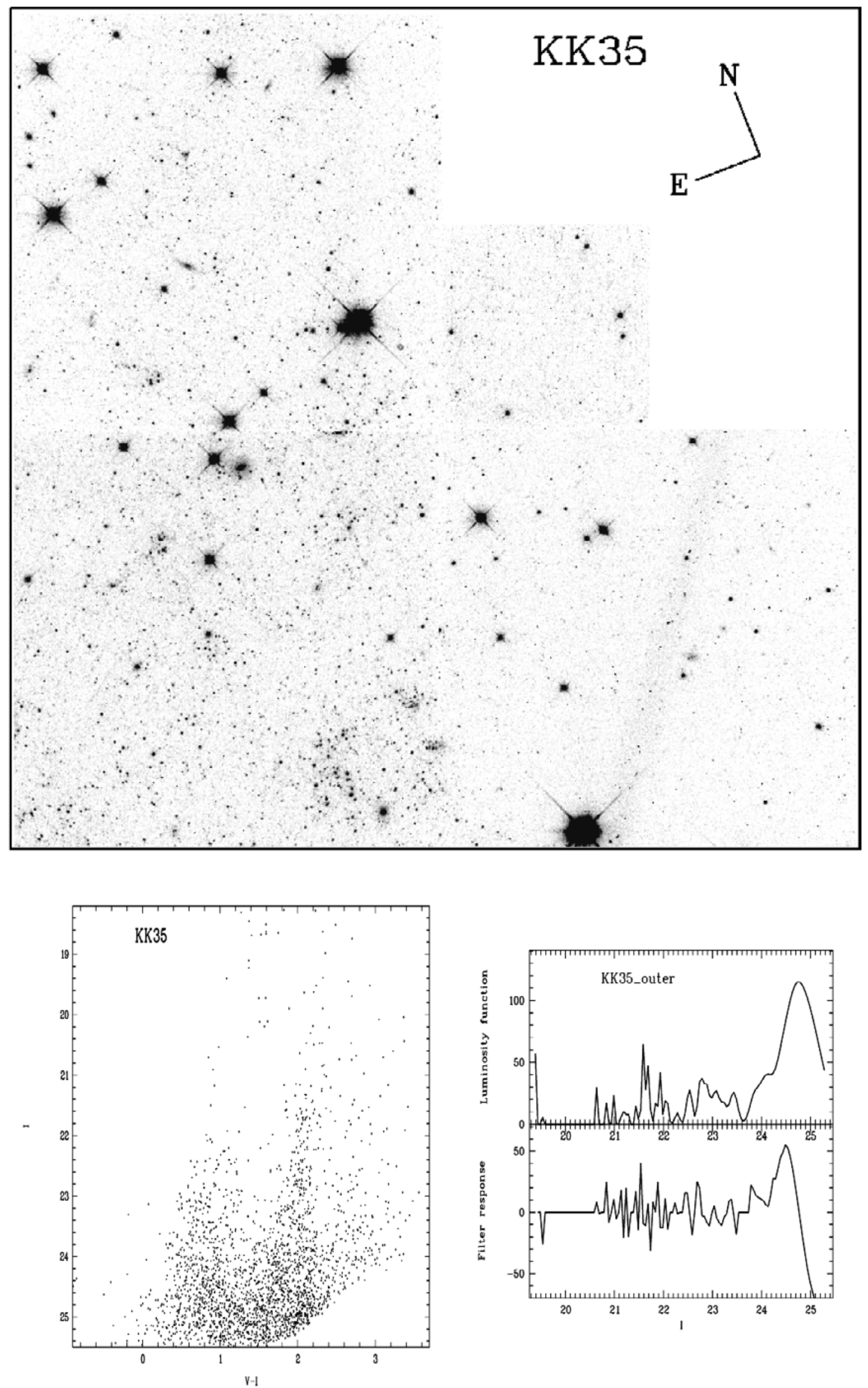

Fig. A.1. continued. 
I. D. Karachentsev et al.: IC 342 group of galaxies, Online Material p 4
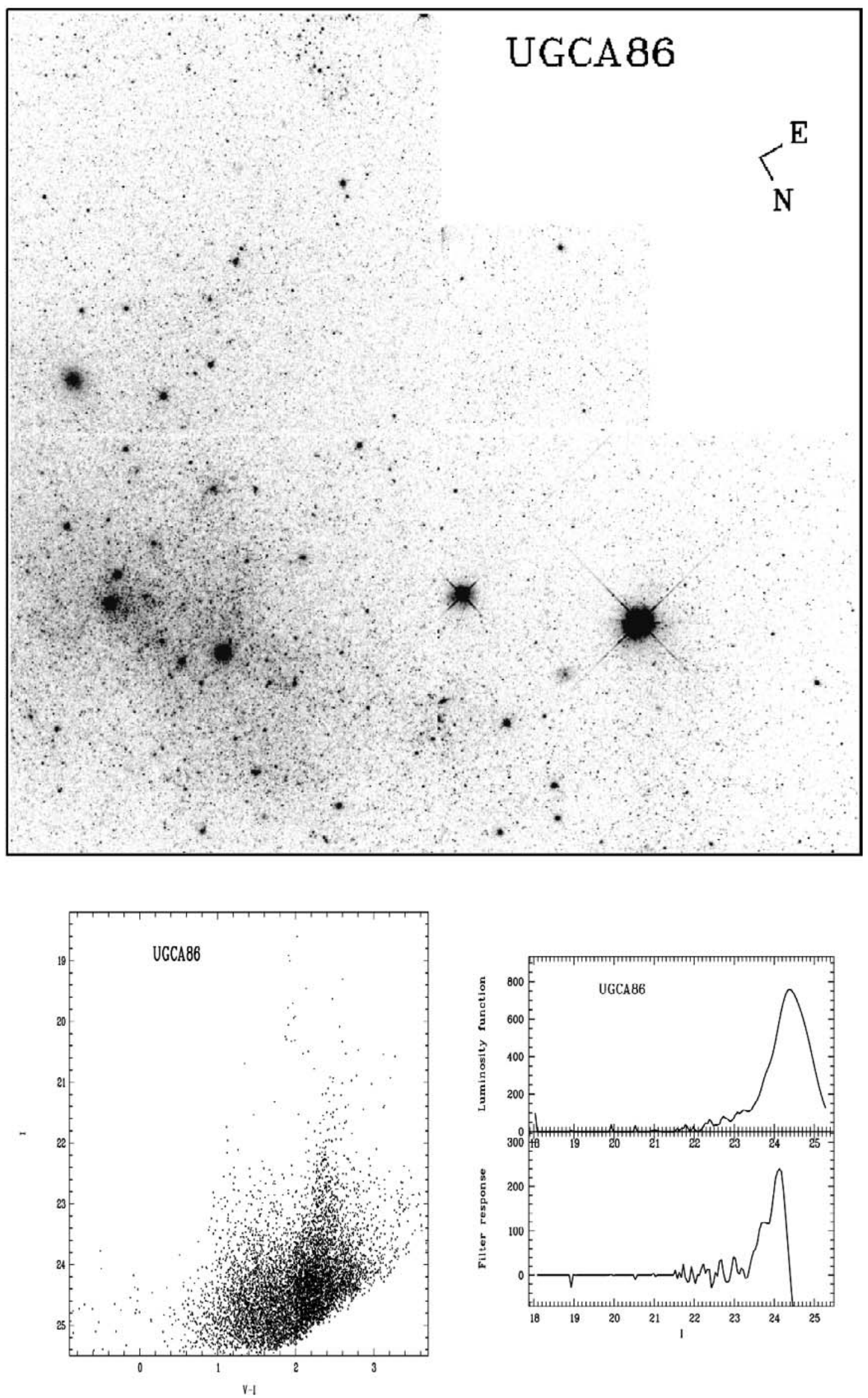

Fig. A.1. continued. 
I. D. Karachentsev et al.: IC 342 group of galaxies, Online Material p 5
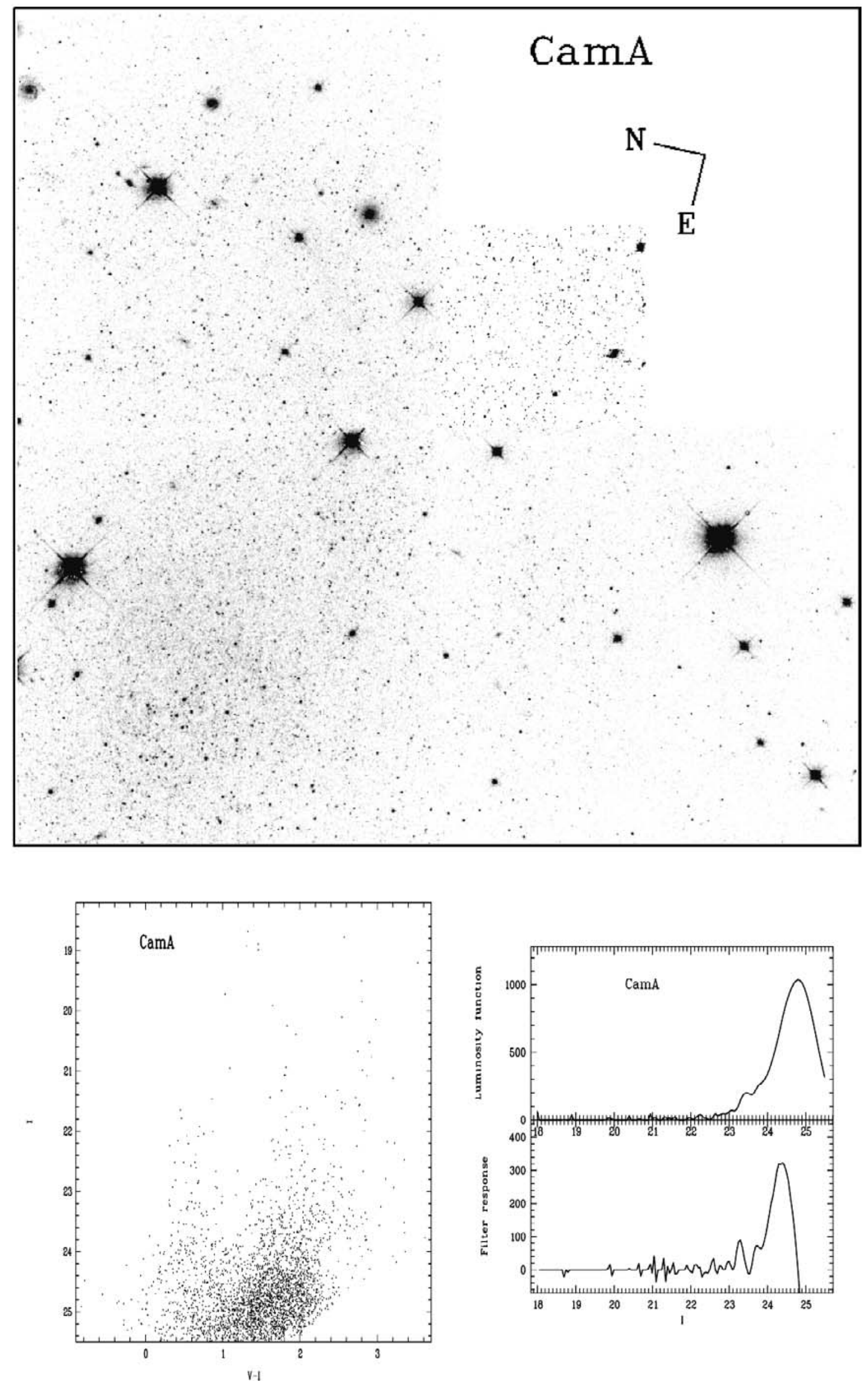

Fig. A.1. continued. 

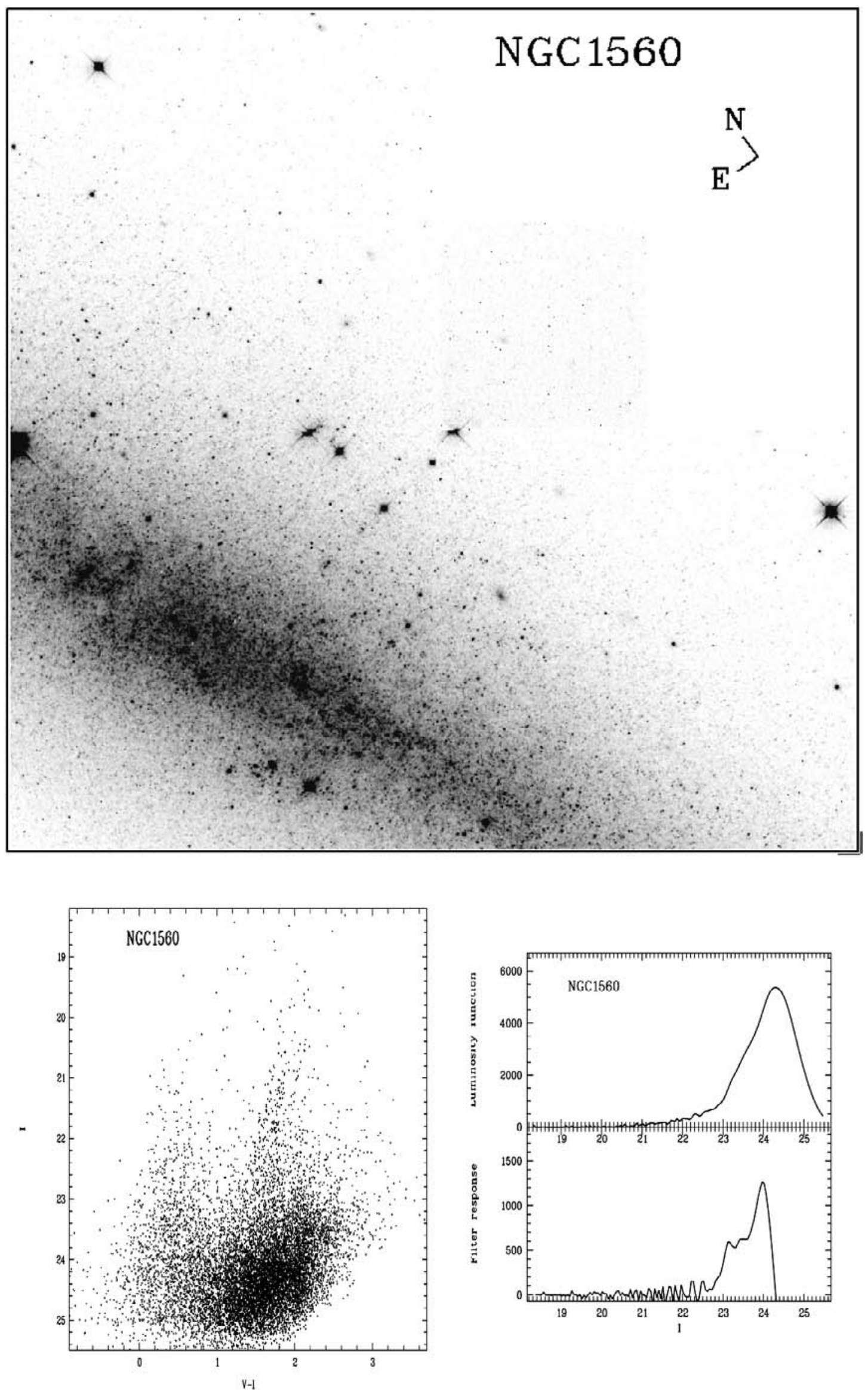

Fig. A.1. continued. 

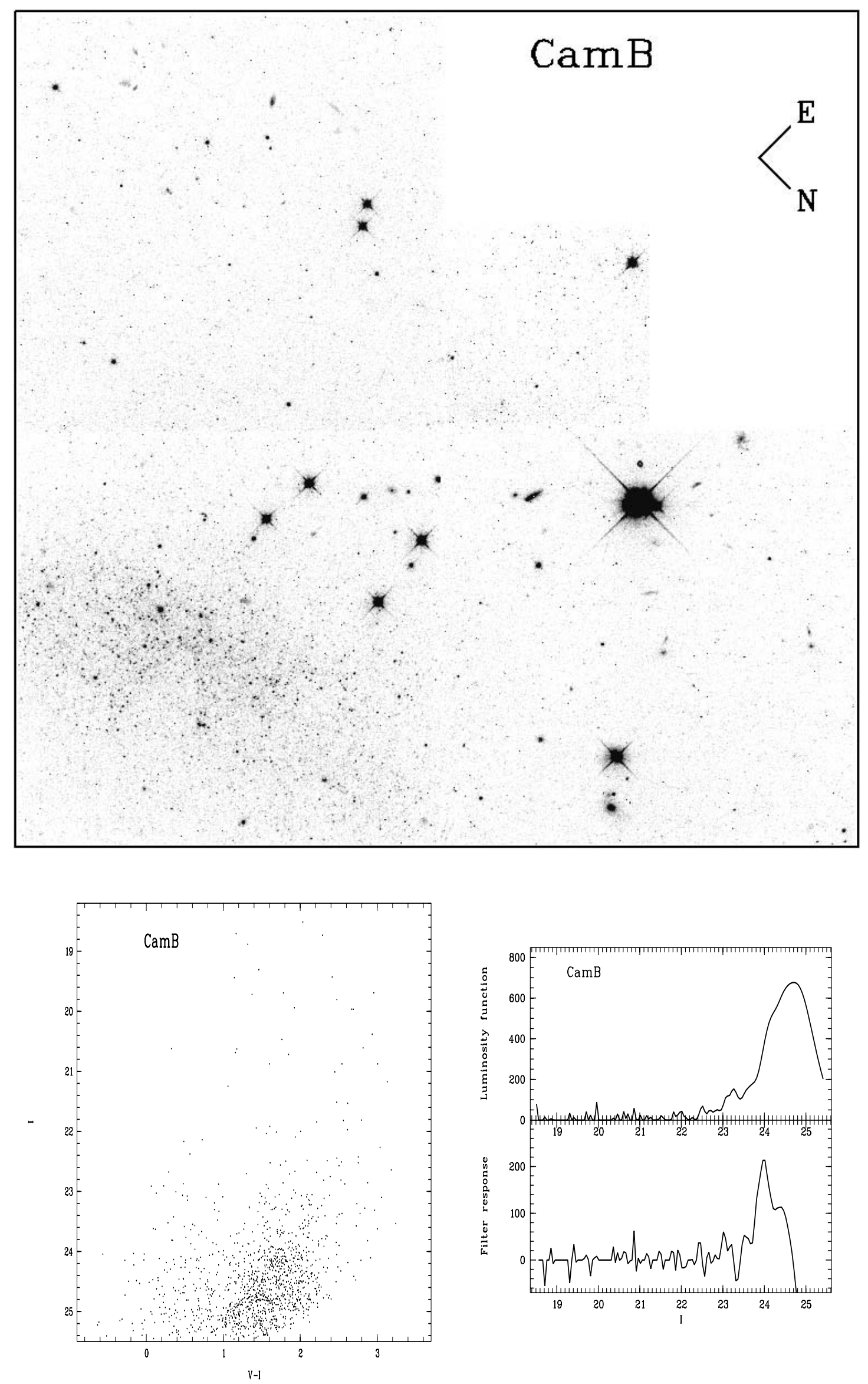

Fig. A.1. continued. 